International Journal of Mathematics for Industry

Vol. 11, No. 1 (2019) 1950003 (11 pages)

(C) The Author(s)

DOI: $10.1142 / \mathrm{S} 2661335219500035$

\title{
A key exchange protocol relying on polynomial maps
}

\author{
Koichiro Akiyama*, ${ }^{\star}$, Shuhei Nakamura ${ }^{\dagger}$, Masaru Ito* and Noriko Hirata-Kohno* \\ *Toshiba Corporation RED Center \\ 1, Komukai-Toshiba-cho, Saiwai-ku, Kawasaki, 212-8582 Kanagawa, Japan \\ ${ }^{\dagger}$ Department of Liberal Arts and Basic Sciences \\ College of Industrial Technology, Nihon University \\ 2-11-1 Shin-ei, Narashino-shi, 275-8576 Chiba, Japan \\ Department of Mathematics \\ College of Science and Technology, Nihon University \\ 1-8-14, Kanda-Surugadai, Chiyoda-ku, 101-8308 Tokyo, Japan \\ \$koichiro.akiyama@toshiba.co.jp
}

Received 26 April 2018

Revised 29 November 2018

Accepted 29 April 2019

Published 7 June 2019

\begin{abstract}
In this paper, we propose a key exchange protocol using multivariate polynomial maps whose security relies on the hardness in finding a solution to a certain system of nonlinear polynomial equations. Under the hardness assumption of solving the system of equations, we prove that our protocol is secure against key recovery attacks by passive attackers if the protocol is established honestly.
\end{abstract}

Keywords: Key exchange protocol; security proof; nonlinear polynomial equation; multivariate equation.

\section{Introduction}

As is well-known, one of the current major problems in cryptography is the study of a post-quantum cryptosystem (PQC for short), resistant to quantum computers. We have candidates of PQC such as lattice-based cryptosystem, code-based cryptosystem and multivariate cryptosystem. The lattice-based cryptosystem and the code-based one yield provably secure schemes including encryption, signature and key exchange, whereas in the multivariate cryptosystem, it seems that very few encryption/key exchange are known to be provably secure so far.

On the other hand, the multivariate cryptosystem is a good promising candidate of PQC, since the security depends on a typical nonlinear problem, e.g., solving multivariate nonlinear equations that is indeed a prominent NP-hard problem. A big distinction between the multivariate cryptosystem and the others is symbolically described by the fact that the security of the latter depends on linear problems, such as the problems of the learning with

This is an Open Access article published by World Scientific Publishing Company. It is distributed under the Creative Commons Attribution 4.0 (CC BY) License. Further distribution of this work is permitted, provided the original work is properly cited. 


\section{K. Akiyama et al.}

errors or the linear decoding problems. Therefore, it is one of the most important issues toward PQC to establish a provably secure multivariate cryptosystem whose security depends on solving multivariate nonlinear equations. It is expected to extend the cryptology itself as well.

In this paper, we propose a provably secure key exchange scheme whose security relies on solving sort of multivariate nonlinear equations. Our scheme is inspired by Yosh's key exchange scheme. ${ }^{9}$

Yosh's scheme is a key exchange protocol which shares an integer (or an element of a finite field) as a secret key using a specific type of polynomial. Since this polynomial is generated by univariate polynomials of the form $(X+a)^{b}+c$ (regarded as secret maps), it becomes of high degree (at least a power of three) which restricts the choice of parameters for the efficient implementation (see 3.1 Observation); in addition, security proof is missing.

In contrast, we build here a provably secure variant of Yosh's scheme employing the secret maps as multivariate polynomial maps, which enable us to overcome drawbacks of Yosh's scheme.

First, by making the secret map multivariate, we can adapt various classes of polynomial maps, e.g., those of lower degree such as quadratic polynomial maps based on the Hidden Field Equations (HFE). The flexibility on the choice of polynomial maps helps to enhance the security level, as is emphasized by providing two examples of polynomial maps (injective and non-injective ones, respectively) which are well applicable in the multivariate publickey cryptography.

Secondly, a security proof is also given against key recovery attacks by passive observers with honest party, under the assumption of the hardness in solving a system of multivariate nonlinear polynomial equations established from the scheme. This scheme is possibly the first secure one with an explicit security proof, among key exchange protocols based on multivariate cryptosystems. It is possible to carry out our security proof in a more general scheme; we also claim that it works for a specific class of polynomial maps (confer Remark 6.5).

The organization of the paper is as follows. Section 2 provides preliminaries and introduction on polynomial maps. In Sec. 3, we briefly review Yosh's key exchange protocol. Section 4 is devoted to introduce our new protocol. In Sec. 5, we give two examples of polynomial maps and discuss prospects for future possibilities. We give in Sec. 6 a security proof of our protocol and observe the security against considerable attacks. We summarize our conclusion in Sec. 7, together with further research programs.

\section{Polynomial Maps}

Let $R$ be a domain. Mainly we let $R=\mathbb{F}_{q}$ be a finite field of $q$ elements. Denote by $R[\underline{x}]=R\left[x_{1}, \ldots, x_{n}\right]$ the polynomial ring of $n$ variables over $R$. A polynomial map is a map $\underline{\psi}=\left(\psi_{1}, \ldots, \psi_{n}\right): R^{n} \rightarrow R^{n}$ of the form

$$
\left(a_{1}, \ldots, a_{n}\right) \mapsto\left(\psi_{1}\left(a_{1}, \ldots, a_{n}\right), \ldots, \psi_{n}\left(a_{1}, \ldots, a_{n}\right)\right),
$$

with $\psi_{1}, \ldots, \psi_{n} \in R[\underline{x}]$. Therefore, a polynomial map is viewed as an element of the Cartesian product $R[\underline{x}]^{n}$. The degree of a polynomial map $\psi=$ $\left(\psi_{1}, \ldots, \psi_{n}\right)$ is defined by

$$
\operatorname{deg} \underline{\psi}=\max \left\{\operatorname{deg} \psi_{1}, \ldots, \operatorname{deg} \psi_{n}\right\},
$$

where $\operatorname{deg} \psi_{j}$ is the total degree of the polynomial $\psi_{j}$ $(1 \leq j \leq n)$. A polynomial map of degree one is called an affine map.

For polynomial maps $\psi=\left(\psi_{1}, \ldots, \psi_{n}\right)$ and $\underline{\varphi}=\left(\varphi_{1}, \ldots, \varphi_{n}\right)$, we define the composed map $\bar{\psi} \circ \underline{\varphi}$ by

$$
\underline{\psi} \circ \underline{\varphi}=\left(\psi_{1}\left(\varphi_{1}, \ldots, \varphi_{n}\right), \ldots, \psi_{n}\left(\varphi_{1}, \ldots, \varphi_{n}\right)\right) .
$$

We also note

$$
\begin{aligned}
& \Lambda_{n, d}:=\left\{f \in R\left[x_{1}, \ldots, x_{n}\right] \mid \operatorname{deg} f=d\right\}, \\
& \left(\Lambda_{n, d}^{n}\right)^{*}:=\left\{\underline{\psi} \in \Lambda_{n, d}^{n} \mid \underline{\psi} \text { is injective }\right\} .
\end{aligned}
$$

\section{Key Exchange Protocol of Yosh}

Yosh $^{9}$ proposed a key exchange protocol based on Diophantine equations. Below we provide a basic description of Yosh's protocol sharing a common key in $\mathbb{Z}$. It is also possible to provide a modification working on a finite field $\mathbb{F}_{p}$ (confer Ref. 6).

Alice and Bob are willing to agree to a secret key using insecure channels for their communications.

(1) Alice chooses an element $\underline{\sigma}\left(\in \mathbb{Z}^{n}\right)$ and constructs a polynomial Diophantine equation with integer coefficients to send to Bob keeping the solution secret.

(a) Generate an integral solution $\underline{\sigma}\left(\in \mathbb{Z}^{n}\right)$ randomly. 
(b) Generate a Diophantine equation with integer coefficients $f(\underline{x})=0$ having $\underline{\sigma}$ as a solution.

(c) Alice sends $f(\underline{x})$ to Bob.

(2) Bob sends polynomials $g(\underline{x})$ and $c(\underline{x})$ with integer coefficients to Alice.

(a) Generate $g(\underline{x}) \in \mathbb{Z}\left[x_{1}, \ldots, x_{n}\right]$ randomly.

(b) Generate a transformation

$$
T_{a_{i}, b_{i}, c_{i}}: X \mapsto\left(X+a_{i}\right)^{b_{i}}+c_{i} \quad(i=1, \ldots, \ell),
$$

randomly, where $a_{i}, c_{i} \in \mathbb{Z}$ and $b_{i}$ is an odd positive integer.

(c) Composing such transformations, compute the polynomial

$$
H(g(\underline{x}))=T_{a_{\ell}, b_{\ell}, c_{\ell}}\left(\ldots\left(T_{a_{1}, b_{1}, c_{1}}(g(\underline{x}))\right) \ldots\right) .
$$

(d) Generate $r(\underline{x}) \in \mathbb{Z}\left[x_{1}, \ldots, x_{n}\right]$ randomly.

(e) Compute the polynomial $c(\underline{x})=H(g(\underline{x}))+$ $f(\underline{x}) r(\underline{x})$.

(f) Bob sends the polynomials $g(\underline{x})$ and $c(\underline{x})$ to Alice.

(3) Alice generates a common key $s \in \mathbb{Z}$ and sends a value $u \in \mathbb{Z}$ to Bob.

(a) Compute $g(\underline{\sigma})=s$ and use it as the common key.

(b) Compute $c(\underline{\sigma})=u$ and send it to Bob.

(4) Bob computes the common key $s$.

Since $f(\underline{\sigma})=0$ implies $c(\underline{\sigma})=H(g(\underline{\sigma}))=u$, Bob can compute the common key $s$ by apply$\operatorname{ing} T_{a_{j}, b_{j}, c_{j}}^{-1}$ to $u$ :

$T_{a_{1}, b_{1}, c_{1}}^{-1}\left(\ldots\left(T_{a_{\ell}, b_{\ell}, c_{\ell}}^{-1}(u)\right) \ldots\right)=H^{-1}(u)=g(\underline{\sigma})=s$.

Note that $T_{a_{j}, b_{j}, c_{j}}^{-1}$ is given by

$$
T_{a_{j}, b_{j}, c_{j}}^{-1}(X)=\left(X-c_{j}\right)^{1 / b_{j}}-a_{j} .
$$

The key exchange protocol by Yosh is summarized in Table 1 , where $\stackrel{r}{\leftarrow}$ implies generating uniformly at random.

\subsection{Observation}

Here we review practical parts of Yosh's protocol to discuss the security.

(a) The key exchange protocol proposed by Yosh uses a particular transformation $T_{a, b, c}(X)=$ $(X+a)^{b}+c$ which owes only three parameters. Then, the major parameter to complicate the
Table 1. Key exchange protocol of Yosh.

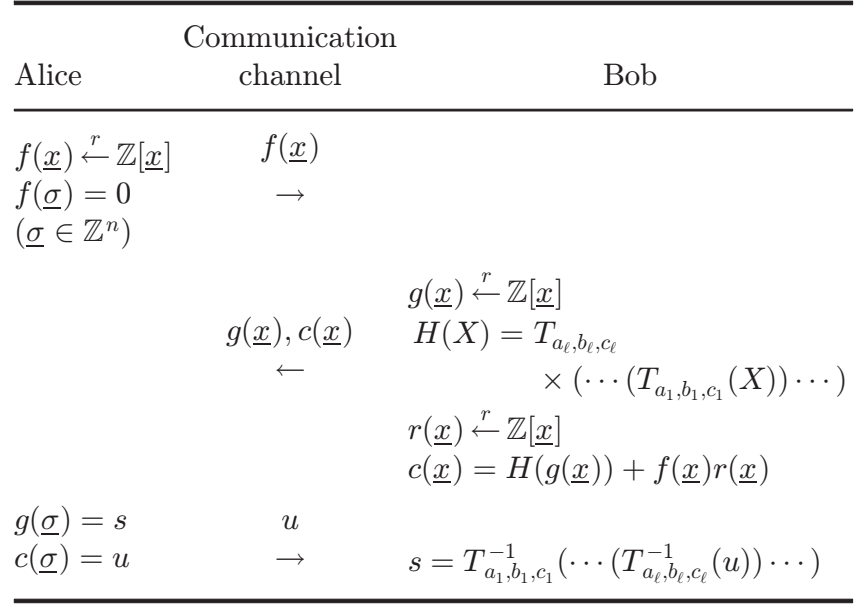

composed map $H(X)$ is the degree $b$ and the number $\ell$ of compositions. However, since the odd integer $b=\operatorname{deg}\left(T_{a, b, c}\right)$ is at least $3(b=1$ is redundant), the degree of the polynomial $c(\underline{x})$ is to be $\geq 3^{\ell}$. Then, the size of data $H(g(\underline{x})$ ) (hence $c(\underline{x})$ ) consequently increases of exponential order. It follows a practical choice of the parameters $b$ and $\ell$ to be restricted, which leads a lack of the security.

(b) Yosh's protocol relies on the hardness in solving the Diophantine equations in integers

$$
\left\{\begin{array}{l}
f\left(x_{1}, \ldots, x_{n}\right)=0 \\
c\left(x_{1}, \ldots, x_{n}\right)=u
\end{array}\right.
$$

whereas no security proof is mentioned. The key exchange protocol proposed in this paper on the other hand provides an explicit security proof under the assumption of hardness in solving a certain system of multivariate nonlinear polynomial equations.

\section{Our New Key Exchange Protocol}

In this section, we propose a new key exchange protocol using polynomial maps. The protocol involves the four parameters $q, n, m, d$ as follows: $q$ is the cardinality of the coefficient field, $n$ is the number of variables, $m$ is the degree of Bob's polynomial map, and $d$ is the degree of Alice's polynomial.

Alice and Bob are going to agree to a common key through an insecure communication channel.

(1) Alice sends a multivariate equation $f(\underline{x})=0$ to Bob keeping a solution $\underline{\sigma} \in \mathbb{F}_{q}^{n}$ secret.

(a) Generate a polynomial $f(\underline{x}) \in \mathbb{F}_{q}\left[x_{1}, \ldots, x_{n}\right]$ of degree $d$ uniformly at random. 
(b) Generate a random solution $\underline{\sigma}\left(\in \mathbb{F}_{q}^{n}\right)$ of $f(\underline{x})=0$ as follows. Choose $\sigma_{1}, \ldots, \sigma_{n-1} \in \mathbb{F}_{q}$ uniformly at random and solve the following univariate polynomial equation:

$$
f\left(\sigma_{1}, \ldots, \sigma_{n-1}, x_{n}\right)=0 \quad \text { in } x_{n} \in \mathbb{F}_{q},
$$

(factorize $f\left(\sigma_{1}, \ldots, \sigma_{n-1}, x_{n}\right)$ in $x_{n} \in \mathbb{F}_{q}$ and find factors of degree one if possible, otherwise modify the constant term and restart the generation).

(c) Keep the solution $\sigma_{n} \in \mathbb{F}_{q}$ to the equation (2).

(d) Send $f(\underline{x})$ to Bob.

(2) Bob sends polynomial maps $g(\underline{x})$ and $\underline{c}(\underline{x})$ to Alice.

(a) Generate a bijective affine map $g(\underline{x})=$ $\left(g_{1}(\underline{x}), \ldots, g_{n}(\underline{x})\right) \in \mathbb{F}_{q}[\underline{x}]^{n} \quad$ uniformly at random.

(b) Generate an injective polynomial map $\underline{\psi}(\underline{x})=\left(\psi_{1}(\underline{x}), \ldots, \psi_{n}(\underline{x})\right) \in \mathbb{F}_{q}[\underline{x}]^{n} \quad$ with $\overline{\operatorname{deg}} \psi_{j}=m(1 \leq j \leq n)$ at random.

(c) Compute $\psi(g(\underline{x}))$.

(d) Generate a polynomial map $\underline{r}(\underline{x})=$ $\left(r_{1}(\underline{x}), \ldots, r_{n}(\underline{x})\right) \in \mathbb{F}_{q}[\underline{x}]^{n}$ of $\operatorname{deg} r_{i}=m-d$ uniformly at random.

(e) Compute the polynomial map $\underline{c}(\underline{x})=$ $\psi(g(\underline{x}))+f(\underline{x}) \underline{r}(\underline{x})$. The term $f(\underline{x}) \underline{r}(\underline{x})$ hides the term $\psi(g(\underline{x}))$; it is expected that almost all terms in $f \underline{r}$ and those in $\underline{\psi}(\underline{g})$ overlap each other.

(f) Send $\underline{g}(\underline{x})$ and $\underline{c}(\underline{x})$ to Alice.

(3) Alice generates a common key $\underline{s} \in \mathbb{F}_{q}^{n}$ to send a value $\underline{u} \in \mathbb{F}_{q}^{n}$ to Bob.

(a) Compute $\underline{g}(\underline{\sigma})=\underline{s}$ and use it as the common key.

Table 2. Our key exchange protocol.

\begin{tabular}{lcl}
\hline Alice & $\begin{array}{c}\text { Communication } \\
\text { channel }\end{array}$ & \multicolumn{1}{c}{ Bob } \\
\hline$f(\underline{x}) \stackrel{r}{\leftarrow} \Lambda_{n, d}$ & $f(\underline{x})$ & \\
$f(\underline{\sigma})=0$ & $\rightarrow$ & \\
$\left(\underline{\sigma} \in \mathbb{F}_{p}^{n}\right)$ & & $\underline{\psi}(\underline{x}) \stackrel{r}{\leftarrow}\left(\Lambda_{n, m}^{n}\right)^{*}$ \\
& $\underline{g}(\underline{x}) \stackrel{r}{\leftarrow} \Lambda_{n, 1}^{n}$ \\
& $\underline{\underline{x}}(\underline{x}) \stackrel{r}{\leftarrow} \Lambda_{n, m-d}^{n}(\underline{x})$ & $\underline{c})=\underline{\psi} \underline{g}(\underline{x}))+f(\underline{x}) \underline{r}(\underline{x})$ \\
& $\leftarrow$ & $\underline{s}=\underline{\psi}^{-1}(\underline{u})$ \\
\hline$\underline{g}(\underline{\sigma})=\underline{s}$ & $\underline{u}$ &
\end{tabular}

(b) Compute $\underline{c}(\underline{\sigma})=\underline{u}$ and send it to Bob.

(4) Bob computes the common key $\underline{s}$.

Since $f(\underline{\sigma})=0$ implies $\underline{c}(\underline{\sigma})=\psi(g(\underline{\sigma}))=\underline{u}$, Bob can compute the common key $\underline{s}$ by applying $\underline{\psi}^{-1}$ to $\underline{u}$ :

$$
\underline{\psi}^{-1}(\underline{u})=\underline{g}(\underline{\sigma})=\underline{s} .
$$

Our proposed key exchange protocol is summarized in Table 2. In Appendix, we show examples of our carried out protocol for understanding.

\subsection{Efficiency}

The efficiency and the security of the protocol are noted as follows:

- The polynomial map $\psi$ should be chosen so that the computation of the inverse $\psi^{-1}(\underline{u})$ in step 4 is efficient. We give in Sec. 5 a few of examples of considerable polynomial maps whose inverse can be calculated in polynomial time.

- In view of the computational efficiency, Bob's steps 2 and 4 will require more processing time than others. Usually, the efficiency of the step 2 depends on the degree of $\psi$ and the one of the step 4 depends on the structure of $\psi$.

- A passive attacker can observe the information $(f(\underline{x}), \underline{c}(\underline{x}), g(\underline{x}), \underline{u})$. Then, one possible attack is to solve the system of multivariate equation

$$
\left\{\begin{array}{c}
f\left(x_{1}, \ldots, x_{n}\right)=0 \\
c_{1}\left(x_{1}, \ldots, x_{n}\right)=u_{1} \\
\vdots \\
c_{n}\left(x_{1}, \ldots, x_{n}\right)=u_{n},
\end{array}\right.
$$

which has the unique solution $\underline{\sigma}$, then the common key $\underline{s}$ can be recovered as $\underline{s}=g(\underline{\sigma})$. It is expected that the polynomial map $\underline{c}(\underline{x})=\underline{\psi} \circ$ $\underline{g}(\underline{x})+f(\underline{x}) \underline{r}(\underline{x})$ sent by Bob is complicated enough and that the system of multivariate equation (3) is hard to solve. This is formulated as Assumption 6.3.

\subsection{Modification of the proposed protocol}

We discuss a modification to make the polynomial map $\psi$ various to enrich the complexity of the proposed key exchange protocol. In Sec. 4, we employ injective polynomial map $\psi$ since Bob needs to recover the unique common key $\underline{s}=\underline{\psi}^{-1}(\underline{u})$. 
Remark that the protocol only requires the invertibility of $\psi$ at $\underline{u}$. Even if this invertibility does not hold, one can try to restart the protocol until the inverse image $\left\{\underline{\psi}^{-1}(\underline{u})\right\}$ becomes a singleton. This approach works when the invertibility of $\psi$ at $\underline{u}$ is of high probability.

Now consider the case when the probability of the invertibility of $\psi$ is not high at $\underline{u}$. Below we propose a modification of our proposed protocol, through the criterion $f(\underline{\sigma})=0$ to exclude noncommon keys.

\subsubsection{Modification}

$$
\text { Put } S=\left\{\underline{\psi}^{-1}(\underline{u}) \mid f\left(\underline{g}^{-1}\left(\underline{\psi}^{-1}(\underline{u})\right)\right)=0\right\} .
$$

In the key exchange protocol proposed in Sec. 4, replace the procedure in Bob's step 4 by the following ones (a)-(d):

(a) Compute all the elements of the set $\left\{\psi^{-1}(\underline{u})\right\}$.

(b) If $\#\left\{\underline{\psi}^{-1}(\underline{u})\right\}=1$, then share the single element $\psi^{-1}(\underline{\bar{u}})$ as a common key.

(c) If $\#\left\{\underline{\psi}^{-1}(\underline{u})\right\}>1$, then compute all the elements of $S$. Namely, pick each element from the set $\left\{\underline{\psi}^{-1}(\underline{u})\right\}$ and verify whether the identity $f\left(\underline{g}^{-1}\left(\underline{\psi}^{-1}(\underline{u})\right)\right)=0$ holds. If it does not hold, then exclude this element from $\left\{\underline{\psi}^{-1}(\underline{u})\right\}$.

(d) If $\# S=1$, then share the single element of $S$ as a common key. Otherwise, retry the procedure of the key exchange from step 1.

Since $\underline{s}=g(\underline{\sigma})$ and $f(\underline{\sigma})=0$, the set $S$ contains the true common key $\underline{s}$. When it occurred $\# S=1$ of desired probability, Alice and Bob can efficiently share the unique element as a common key. In Sec. 5.2, an example of this modification is given.

\subsection{Comparison with Yosh's protocol}

We conclude this section with remarks on the difference with Yosh's protocol.

Yosh's protocol uses a single polynomial for $c(\underline{x})$ while we use $\left(c_{1}(\underline{x}), \ldots, c_{n}(\underline{x})\right)$. Therefore, the shared common key of our protocol is $n$ times longer than the Yosh's one (working on a finite filed). This also means that the time and the space complexity of our protocol can be at least $n$ times larger. However, the actual complexity comparison is not so clear in practice, because it highly depends on the parameter choice or the structure of $\underline{\psi}$.
We can adapt various classes of multivariate polynomial maps in the choice of $\psi$ to enhance the efficiency and the security, while Yosh's protocol employs a specific kind of the univariate polynomial $T_{a, b, c}$. In particular, as in Sec. 5.2, we can efficiently employ quadratic polynomial maps based on the HFEs, whereas Yosh's protocol uses a polynomial $c(\underline{x})$ of degree at least $3^{\ell}$. In other words, we can enhance the security level with the number of variables keeping degree to be lower, in contrast to Yosh's protocol whose security is enhanced by the number of composition leading exponential growth of degree (confer 3.1 Observation (a)).

\section{Generation of the Polynomial $\operatorname{Map} \underline{\psi}$}

In this section, we give two concrete examples of generating the polynomial map $\underline{\psi}$ as is proposed in Sec. 4. We introduce invertible polynomial maps and the HFE-based polynomial maps, which are frequently adapted in the multivariate public-key cryptography. ${ }^{3}$

We remark that these two are injective and noninjective, respectively. Therefore, we can employ the invertible polynomial maps in our protocol while the applicability of the HFE-based ones is not clear. We give in Sec. 5.2.1 an experimental result to show that the modification of our protocol can be efficiently applied to the HFE-based ones.

\subsection{Invertible polynomial map}

A polynomial map $\underline{\rho}=\left(\rho_{1}, \ldots, \rho_{n}\right) \in R[\underline{x}]^{n}$ is said to be invertible if there exists a polynomial map $\underline{\tau}=\left(\tau_{1}, \ldots, \tau_{n}\right) \in R[\underline{x}]^{n}$ such that

$$
\underline{\rho} \circ \underline{\tau}=\left(x_{1}, \ldots, x_{n}\right) \text {. }
$$

We call $\underline{\tau}=\rho^{-1}$ the inverse of $\rho$.

We construct invertible polynomial maps $\underline{\varphi}_{1}, \ldots, \underline{\varphi}_{\ell}$ and define the polynomial map $\underline{\psi}$ by

$$
\underline{\psi}=\underline{\varphi}_{1} \circ \cdots \circ \underline{\varphi}_{\ell} .
$$

Here, each $\underline{\varphi}_{i}$ has an inverse supposed to be easily computed. The inverse of $\underline{\psi}$ is given by

$$
\underline{\psi}^{-1}=\underline{\varphi}_{\ell}^{-1} \circ \cdots \circ \underline{\varphi}_{1}^{-1} .
$$

Now let us focus on the invertible polynomial map $\underline{\psi}$ which admits an explicit inverse expression. 
An elementary polynomial map is a polynomial map of the form

$$
\begin{aligned}
\underline{\psi}(\underline{x})= & \left(x_{1}, \ldots, x_{i-1}, x_{i}+g\left(x_{1}, \ldots, x_{i-1},\right.\right. \\
& \left.\left.x_{i+1}, \ldots, x_{n}\right), x_{i+1}, \ldots, x_{n}\right),
\end{aligned}
$$

with $g \in R\left[x_{1}, \ldots, x_{i-1}, x_{i+1}, \ldots, x_{n}\right]$. The inverse of $\underline{\psi}$ is given by

$$
\begin{aligned}
\underline{\psi}^{-1}(\underline{x})= & \left(x_{1}, \ldots, x_{i-1}, x_{i}-g\left(x_{1}, \ldots, x_{i-1},\right.\right. \\
& \left.\left.x_{i+1}, \ldots, x_{n}\right), x_{i+1}, \ldots, x_{n}\right) .
\end{aligned}
$$

A polynomial map is said to be tame if it is expressed by a composition of elementary polynomial maps and invertible affine maps. A typical example of tame polynomial maps is called triangular polynomial map, which is indeed of the form

$$
\begin{aligned}
\underline{\psi}(\underline{x})= & \left(x_{1}+g_{1}, x_{2}+g_{2}\left(x_{1}\right), \ldots, x_{n}\right. \\
& \left.+g_{n}\left(x_{1}, \ldots, x_{n-1}\right)\right)
\end{aligned}
$$

with $g_{1} \in R, g_{i} \in R\left[x_{1}, \ldots, x_{i-1}\right](i \geq 2)$. Note that this polynomial map is expressed by the composition of elementary polynomial maps as follows:

$$
\begin{aligned}
\underline{\psi}= & \underline{\varphi}_{1} \circ \cdots \circ \underline{\varphi}_{n} \\
\text { with } \underline{\varphi}_{i}(\underline{x})= & \left(x_{1}, \ldots, x_{i-1}, x_{i}+g_{i}\left(x_{1}, \ldots, x_{i-1}\right),\right. \\
& \left.x_{i+1}, \ldots, x_{n}\right) .
\end{aligned}
$$

In view of the formula (6), the inverse is efficiently computable by the substitution to (5). $\mathrm{Moh}^{7}$ proposed to use triangular polynomial maps in public-key cryptosystem, in so-called Tame Transformations Method (TTM).

Since invertible polynomial maps are injective, we can adapt the above examples of polynomial maps for our proposed protocol. Then, step 4 will be efficient because this is done by substitution for (5). The efficiency of step 2 depends on how $\operatorname{deg} \psi$ is small, trading-off with the security level.

\subsection{Hidden field equations based polynomial maps}

Let us consider the HFE-based cryptosystem which yields non-injective polynomial maps. Let $K=$ $\mathbb{F}_{q}\left[\theta_{1}, \ldots, \theta_{n}\right]$ be an extension of $\mathbb{F}_{q}$ of degree $n$, where the elements $\theta_{1}, \ldots, \theta_{n}$ form a basis of $K$ over $\mathbb{F}_{q}$. An HFE-based polynomial map $\underline{\psi}$ is generated by

$$
\underline{\tilde{\psi}}(\underline{x})=\phi^{-1} \circ F \circ \phi(\underline{x}) .
$$

Here, the map $\phi: \mathbb{F}_{q}^{n} \rightarrow K$ is an induced isomorphism $\phi\left(x_{1}, \ldots, x_{n}\right)=x_{1} \theta_{1}+\cdots+x_{n} \theta_{n}$ and $F \in$ $K[X]$ is a univariate polynomial of the following form (the summation is taken over non-negative integers $i, j$ so that $\operatorname{deg} F \leq D$ with a given $D$ ):

$$
F(X)=\sum_{i, j} \alpha_{i j} X^{q^{i}+q^{j}}+\sum_{i} \beta_{i} X^{q^{i}}+\gamma,
$$

with $\alpha_{i j}, \beta_{i}, \gamma \in K$. Consider the following construction of the polynomial map $\underline{\psi}$ based on the HFE:

$$
\begin{aligned}
& \underline{\psi}(\underline{x})=L_{2} \circ \underline{\tilde{\psi}} \circ L_{1}(\underline{x}), \\
& L_{1}, L_{2}: \text { bijective affine maps on } \mathbb{F}_{q}^{n} .
\end{aligned}
$$

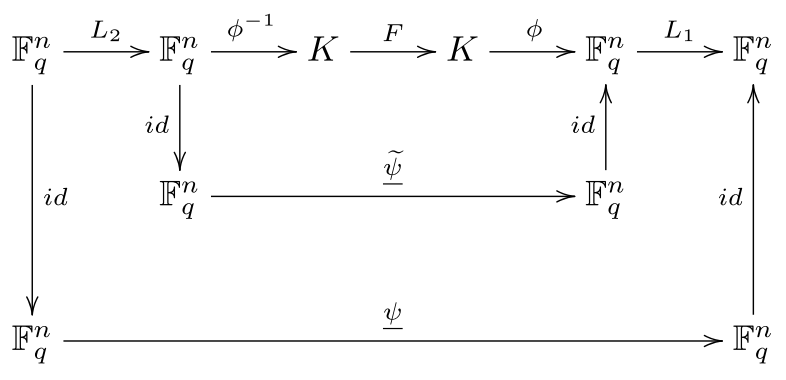

In this case, we have $\operatorname{deg} \psi=2$. Therefore, the possible parameters $(m, d)$ of our protocol is $(m, d)=(2,1)$ or $(2,2)$ (here we recall that $m$ is the degree of the polynomial map $\psi$, see Sec. 4).

The inverse of $\psi$ can be computed in polynomial time by, e.g., Berlekamp's algorithm whose complexity can be estimated as (confer Refs. 2 and 3)

$$
O\left(n D^{2} \log D+D^{3}\right) .
$$

In contrast to the invertible polynomial maps, the HFE-based polynomial map is not injective in general. In this case, we can consider to employ them in the proposed protocol using the modification given in Sec. 4.2. Then step 2 can be efficiently executed. In view of (9), the efficiency of the step 4 depends on $D$ which will have a trade-off with the security level. We also need to ensure, for the efficiency, that one execution of the key exchange has high success rate; we observe this next. 


\subsubsection{Evaluation of the success rate of the protocol with the HFE-based polynomial maps}

Here we examine the HFE-based polynomial map to show parameter settings of high success rate.

We begin with testing random instances of the proposed key exchange protocol with $d=\operatorname{deg} f=1$ using the HFE-based polynomial map $\psi$ in (8). Each test is associated with parameters $(q, n, D)$ where $q$ is the cardinality of the coefficient field, $n$ is the number of variables and $D$ is the degree of the polynomial $F$ defined by $(7)$. For each $(q, n, D)$, we examine $N=300$ random samples of the protocol and compute the rates of samples for each cardinality $\#\left\{\underline{\psi}^{-1}(\underline{u})\right\}$;

$$
\begin{aligned}
& c_{k}(q, n, D) \\
& =\frac{\text { the number of samples in which } \#\left\{\underline{\psi}^{-1}(\underline{u})\right\}=k}{N}
\end{aligned}
$$

and the success rate

$$
\begin{aligned}
& s(q, n, D) \\
& =\frac{\text { the number of samples in which } \# S=1}{N},
\end{aligned}
$$

that is the ratio of samples for which the unique solutions were obtained by using the modification in Sec. 4.2. We test all parameters $(q, n, D)$ from the set $P$ defined by

$$
P=\left\{\begin{aligned}
& q \in\{2,3, \ldots, 9\}, \\
&(q, n, D): n \in\{5,10,15, \ldots, 50\}, \\
& 1 \leq D \leq 1024, D=2 q^{d}, d \in \mathbb{N}
\end{aligned}\right\} .
$$

First, we show Table 3 to see, without our modification, that the success rate of our protocol is not satisfactory. In Table 3 , the column $k=1,2,3,4$ (resp., ' $\geq 5$ ') shows the ratio of the test samples (among $N=300$ tests) for which the cardinality of $\left\{\psi^{-1}(\underline{u})\right\}$ becomes $k$ (resp., more than five). Therefore, the first column represents the success

Table 3. Distribution of the cardinality of $\left\{\underline{\psi}^{-1}(\underline{u})\right\}$.

\begin{tabular}{lccrrr}
\hline & \multicolumn{5}{c}{$\# \underline{\psi}^{-1}(\underline{u})$} \\
\cline { 2 - 6 }$(q, n, D)$ & 1 & 2 & \multicolumn{1}{c}{3} & \multicolumn{1}{c}{4} & $\geq 5$ \\
\hline$(5,5,50)$ & $36.3 \%$ & $37.0 \%$ & $20.3 \%$ & $4.7 \%$ & $1.7 \%$ \\
$(2,50,4)$ & $29.0 \%$ & $53.7 \%$ & $0.0 \%$ & $17.3 \%$ & $0.0 \%$ \\
$(4,25,8)$ & $44.7 \%$ & $30.7 \%$ & $16.3 \%$ & $6.0 \%$ & $2.3 \%$ \\
\hline
\end{tabular}

rate for the corresponding parameter. Instead of listing all parameters $(q, n, D) \in P$ in rows of the table, we only pick the cases $(q, n, D)=(5,5,50)$, $(2,50,4)$, and $(4,25,8)$ corresponding to the cases where $c_{1}(q, n, D)$ (namely, the first column) matches the median, the minimum, and the maximum among all the samples $\left\{c_{1}(q, n, D) \mid(q, n, D) \in P\right\}$, respectively. Note that the average of $c_{1}(q, n, D)$ among all the samples is $36.5 \%$ with the sample standard deviation 0.028. In our experiment, therefore, the protocol without our modification has the success rate less than $50 \%$.

Secondly, let us see the success rate of our protocol using our modification in Sec. 4.2. Table 4 summarizes the distribution of the success rate $s(q, n, D)$ for each $q$ (with $f(\underline{x})$ of degree one). That is, for each fixed $q \in\{2,3, \ldots, 9\}$, we calculated the minimum, the maximum, the average, and the sample standard derivation of the set

$$
\{s(q, n, D) \mid(q, n, D) \in P\},
$$

where the values are converted in percentage form for the columns min, max, and ave. Table 4 shows that our modification fairly works when $q$ is moderately large. Recall that $S$ is the intersection of $\left\{\underline{\psi}^{-1}(\underline{u})\right\}$ and the set of zeroes of $f(\underline{x})$. The proportion of the elements of $S$ in $\mathbb{F}_{q}^{n}$ is at most $1 / q$, for $\#\{$ zeroes of $f(\underline{x})\} \leq q^{n-1}$. Table 3 shows $\#\left\{\underline{\psi}^{-1}(\underline{u})\right\} \leq 4$ of high probability in most of the samples, that leads us to expect that the success rate increases as $q$ (confer Ref. 8 for $f(\underline{x})$ of higher degree).

This experimental result shows that we can efficiently adapt the HFE-based polynomial maps in our protocol if $q$ is appropriately chosen. Then, we select the remaining parameter $(n, D)$ tuning the security level.

Table 4. Distribution of the success rate $s(q, n, D)$. 'sd' shows the sample standard deviation.

\begin{tabular}{ccccc}
\hline$q$ & $\min$ & $\max$ & ave & sd \\
\hline 2 & $54.3 \%$ & $67.0 \%$ & $60.7 \%$ & 0.027 \\
3 & $63.0 \%$ & $78.7 \%$ & $71.4 \%$ & 0.032 \\
4 & $72.3 \%$ & $84.0 \%$ & $77.9 \%$ & 0.022 \\
5 & $73.0 \%$ & $86.7 \%$ & $81.8 \%$ & 0.029 \\
7 & $82.3 \%$ & $89.7 \%$ & $86.7 \%$ & 0.017 \\
8 & $85.0 \%$ & $91.0 \%$ & $87.6 \%$ & 0.016 \\
9 & $86.0 \%$ & $95.0 \%$ & $89.9 \%$ & 0.021 \\
\hline
\end{tabular}




\section{Security of the Protocol}

In this section, we obverse the security of our key exchange protocol.

\subsection{Security proof}

We introduce a cryptographic hardness assumption of solving a certain system of multivariate nonlinear equations. Then under this assumption, we prove that our key exchange protocol is secure in the sense of the security model defined below.

We only describe the security against the Key Recovery Attack by Honest Passive Observer (KRA-HPO). Namely, the attacker does not interfere with the communication between Alice and Bob. To formulate the security model, we define the experiment $\operatorname{Exp}_{\Sigma \mathscr{A}}^{\mathrm{KRA}-\mathrm{HPO}}(k)$ of a key recovery attack $\mathscr{A}$ against our key exchange protocol $\Sigma$ with security parameter $k$. Here $\operatorname{Gen}\left(1^{k}\right)$ is a function that returns parameters ensuring the security parameter $k$.

$$
\begin{aligned}
& \frac{\Sigma(q, n, m, d)}{f \stackrel{r}{\leftarrow} \Lambda_{n, d},} \\
& f(\underline{\sigma})=0, \underline{\sigma} \stackrel{r}{\leftarrow} \mathbb{F}_{q}^{n} ; \\
& \underline{g} \stackrel{r}{\leftarrow} \Lambda_{n, 1}^{n} \\
& \underline{r} \stackrel{r}{\leftarrow} \Lambda_{n, m-d}^{n} ; \\
& \underline{c} \leftarrow \underline{\psi}(\underline{g})+f \cdot \underline{r}, \\
& \underline{\psi} \stackrel{r}{\leftarrow}\left(\Lambda_{n, m}^{n}\right)^{*} ; \\
& \underline{u} \leftarrow \underline{c}(\underline{\sigma}) ; \\
& \underline{s} \leftarrow \underline{\psi}^{-1}(\underline{u}) ; \\
& \text { Output }(\underline{s}, f, \underline{g}, \underline{c}, \underline{u}) \\
& \begin{array}{l}
\frac{\operatorname{Exp}_{\Sigma, \mathscr{A}}^{\mathrm{KRA}-\mathrm{HPO}}(k)}{(q, n, m, d) \stackrel{r}{\leftarrow} \operatorname{Gen}\left(1^{k}\right) ;} \\
(\underline{s}, f, \underline{g}, \underline{c}, \underline{u}) \stackrel{r}{\leftarrow} \Sigma(q, n, m, d) ; \\
\underline{s}^{\prime} \leftarrow \mathscr{A}(f, \underline{g}, \underline{c}, \underline{u}) ; \\
\text { Output }\left(\underline{s}, \underline{s}^{\prime}\right) .
\end{array}
\end{aligned}
$$

Definition 6.1. We say that the protocol $\Sigma$ is secure in the sense of KRA-HPO if

$$
\begin{aligned}
& \operatorname{Adv}_{\Sigma, \mathscr{A}}^{\mathrm{KRA}-\mathrm{HPO}}(k) \\
& \quad=\operatorname{Pr}\left[\underline{s}=\underline{s}^{\prime} \mid\left(\underline{s}, \underline{s}^{\prime}\right) \leftarrow \operatorname{Exp}_{\Sigma, \mathscr{A}}^{\mathrm{KRA}-\mathrm{HPO}}(k)\right],
\end{aligned}
$$

is less than $\varepsilon(k)$ for any probabilistic polynomial time algorithms $\mathscr{A}$ against the protocol $\Sigma$, where $k$ is a security parameter and $\varepsilon$ is a negligible function in the security parameter $k$.
Definition 6.2. The Polynomial Map Equation (PME) problem is a problem of finding a solution to the system of multivariate equations

$$
\left\{\begin{array}{c}
f\left(x_{1}, \ldots, x_{n}\right)=0 \\
c_{1}\left(x_{1}, \ldots, x_{n}\right)=u_{1} \\
\vdots \\
c_{n}\left(x_{1}, \ldots, x_{n}\right)=u_{n}
\end{array}\right.
$$

with the input $(f, \underline{g}, \underline{c}, \underline{u})$ observed by $\Sigma$. Namely, the input is the restriction of the output of $\Sigma$ removing the common key $\underline{s}$.

Note that the PME problem (10) has a unique solution because the polynomial map $\psi$ in the protocol is injective. The solution $\underline{\sigma} \in \mathbb{F}_{q}^{n}$ recovers the common key $\underline{s}$ of the protocol $\Sigma$ by computing $\underline{g}(\underline{\sigma})=\underline{s}$.

Here, we introduce a hardness assumption of solving the PME problem. Consider the experiment $\operatorname{Exp}_{\mathscr{B}}^{\mathrm{PME}}(k)$ of the PME problem for a probabilistic algorithm $\mathscr{B}$ as follows:

$$
\begin{aligned}
& \frac{\operatorname{Exp}_{\mathscr{B}}^{\mathrm{PME}}(k)}{(q, n, m, d) \stackrel{r}{\leftarrow} G e n\left(1^{k}\right) ;} \\
& (\underline{s}, f, \underline{g}, \underline{c}, \underline{u}) \stackrel{r}{\leftarrow} \Sigma(q, n, m, d) ; \\
& \underline{\sigma} \leftarrow \mathscr{B}(f, \underline{g}, \underline{c}, \underline{u}) ; \\
& \text { Output } 1 \text { if } \underline{\sigma} \text { is a solution of }(10) \\
& \text { or else output } 0 .
\end{aligned}
$$

Assumption 6.3 (PME assumption). The advantage

$$
\operatorname{Adv}_{\mathscr{B}}^{\mathrm{PME}}(k):=\operatorname{Pr}\left[r=1 \mid r \leftarrow \operatorname{Exp}_{\mathscr{B}}^{\mathrm{PME}}(k)\right],
$$

is less than $\varepsilon(k)$ for all probabilistic polynomial time algorithms $\mathscr{B}$ of solving the PME problem, where $k$ is a security parameter and $\varepsilon$ is a negligible function in the security parameter $k$.

Now we provide a security estimate of our protocol. The following theorem means that the hardness in breaking the proposed protocol is reducible to the one in solving the PME problem.

Theorem 6.4. Under the PME assumption, the key exchange scheme $\Sigma$ is secure in the sense of KRAHPO. In particular, if there is a probabilistic polynomial time algorithm $\mathscr{A}$ that breaks the scheme $\Sigma$ in the sense of KRA-HPO, then there exists an algorithm $\mathscr{B}$ that solves the PME problem in probabilistic polynomial time such that $\operatorname{Adv}_{\mathscr{B}}^{\mathrm{PME}}(k)=$ $\operatorname{Adv}_{\Sigma, \mathscr{A}}^{\mathrm{KRA}-\mathrm{HPO}}(k)$. 
Proof. Let $\mathscr{A}$ be a probabilistic polynomial time algorithm against the protocol $\Sigma$. Define the algorithm $\mathscr{B}(f, \underline{g}, \underline{c}, \underline{u})$ as follows:

$$
\underline{s}^{\prime} \leftarrow \mathscr{A}(f, \underline{g}, \underline{c}, \underline{u}) ; \quad \underline{\sigma}^{\prime} \leftarrow \underline{g}^{-1}\left(\underline{s}^{\prime}\right) ; \quad \text { Output } \underline{\sigma}^{\prime} .
$$

Note that $\mathscr{B}$ is a polynomial time algorithm since $g$ is a bijective affine map and so $\underline{g}^{-1}\left(\underline{s}^{\prime}\right)$ can be computed in $O\left(n^{3}\right)$.

To prove the identity

$$
\operatorname{Adv}_{\mathscr{B}}^{\mathrm{PME}}(k)=\operatorname{Adv}_{\Sigma, \mathscr{A}}^{\mathrm{KRA}-\mathrm{HPO}}(k),
$$

it suffices to show that, for any input $(f, g, \underline{c}, \underline{u})$, the algorithm $\mathscr{A}(f, \underline{g}, \underline{c}, \underline{u})$ recovers the common key $\underline{s}$ correctly if and only if $\mathscr{B}(f, \underline{g}, \underline{c}, \underline{u})$ outputs the solution $\sigma$ of the PME problem.

Let $(\underline{s}, f, \underline{g}, \underline{c}, \underline{u})$ be an output of the protocol $\Sigma$. Namely, $\underline{s}$ is a common key shared by $\Sigma$ communicating $(f, g, \underline{c}, \underline{u})$. By the definition of the protocol $\Sigma$, there exists $\underline{\sigma} \in \mathbb{F}_{q}^{n}$ such that

$$
f(\underline{\sigma})=0, \quad \underline{c}(\underline{\sigma})=\underline{u}, \quad \underline{s}=\underline{g}(\underline{\sigma}) .
$$

The $\underline{\sigma}$ is the unique solution of the PME problem.

Note that the outputs $\underline{s}^{\prime} \leftarrow \mathscr{A}(f, \underline{g}, \underline{c}, \underline{u})$ and $\underline{\sigma}^{\prime} \leftarrow \mathscr{B}(f, \underline{g}, \underline{c}, \underline{u})$ satisfy $\underline{s}^{\prime}=\underline{g}\left(\underline{\sigma}^{\prime}\right)$.

Suppose that $\mathscr{A}$ recovers the common key correctly, that is, $\underline{s}=\underline{s}^{\prime}$ holds. Then we have $\underline{\sigma}=\underline{g}^{-1}(\underline{s})=\underline{g}^{-1}\left(\underline{s}^{\prime}\right)=\underline{\sigma}^{\prime}$. This implies that the output of $\mathscr{B}$ is the solution of the PME problem.

Conversely, if $\mathscr{B}$ outputs the solution of the PME problem, then we have $\underline{\sigma}=\underline{\sigma}^{\prime}$ and thus $\underline{s}=g(\underline{\sigma})=$ $g\left(\underline{\sigma}^{\prime}\right)=\underline{s}^{\prime}$ holds, showing that the output of $\mathscr{A}$ is the true common key.

Consequently, we obtain (11). The proof is completed.

Remark 6.5. (1) The assumption that $\underline{g}(\underline{x})$ is an affine map is critical for our security proof. More precisely, it relies on the polynomial time invertibility of $\underline{g}(\underline{x})$.

(2) In practice, we restrict to a certain class, say $\mathscr{F}$, of polynomial maps as in Sec. 5, from which $\psi$ is generated. In this case, it is not clear whether Theorem 6.4 is applicable. The same statement of Theorem 6.4 nevertheless holds with the restriction of the class $\left(\Lambda_{n, m}^{n}\right)^{*}$ to the particular class $\mathscr{F}$ in the definition of $\Sigma(q, n, m, d)$.

(3) The above security proof does hold when we employ the modification considered in Sec. 4.2 by a similar argument.

\subsection{Security against attacks}

Here we discuss the security of our protocol against considerable attacks.

\subsubsection{Intractability of the PME problem}

As viewed in Sec. 4, a possible attack against our protocol is solving the system of multivariate equations (10) for which we assumed its hardness as the PME assumption. In general, the PME assumption is connected to the fact that the problem of solving a system of multivariate nonlinear polynomial equations which is NP-hard even if it is quadratic. ${ }^{5}$ The hardness of the PME problem will depend on the class (say $\mathscr{F}$ ) of polynomial map from which $\psi$ is generated. To ensure the security against the attack of solving the PME problem, the class $\mathscr{F}$ (or the parameters behind the class) should be carefully chosen.

\subsubsection{Linear algebra attack}

Since the PME problem is focused on a special type of a system of polynomial equations with an additional information $\underline{g}(\underline{x})$, we also need to observe the possibility of structural attacks to our protocol.

Suppose that the attacker obtained the polynomial map $\psi$ used in the protocol $\Sigma$. Then, the inverse $\underline{s}=\bar{\psi}^{-1}(\underline{u})$ might be efficiently computable. When we employ invertible polynomial maps, there is an inverse formula using the exponential map of a derivation, available in the case of characteristic zero or of sufficiently large prime. ${ }^{4}$ It is also possible to compute the inverse polynomial map in an arbitrary field using Gröbner basis computation. ${ }^{4}$

Therefore, we focus on the attack to recover the secret polynomial map $\psi$. The common key can be computed as $\underline{s}=\underline{\psi}^{-1}(\underline{u})$.

We discuss a considerable attack, the linear algebra attack, of solving a system of linear equations to determine the coefficients of $\psi$ and $\underline{r}$. As far as we know, this is the most practical method to recover the polynomial map $\psi$.

Recall that, in the proposed protocol, the identities

$$
\psi_{i}(\underline{g}(\underline{x}))+f(\underline{x}) r_{i}(\underline{x})=c_{i}(\underline{x}), \quad i=1, \ldots, n,
$$

hold. Letting all possible coefficients of $\psi_{i}(\underline{x})$ and $r_{i}(\underline{x})$ be unknowns, (12) is viewed as a system of linear equations. Since $\operatorname{deg} c_{i}=\operatorname{deg} \psi_{i}=m$ and 
$\operatorname{deg} r_{i}=m-d$, the number of unknowns is $N(n, m)+N(n, m-d)$ and the number of equations is at most $N(n, m)$, where

$$
N(n, d):=n\left(\begin{array}{c}
n+d \\
d
\end{array}\right)
$$

which is the maximal number of terms of polynomial maps in $n$ variables and of degree $d$. Then, the dimension of the solutions of (12) is at least $N(n, m-d)$. Therefore, choosing parameters $(q, n$, $m, d)$ so that $q^{N(n, m-d)}>2^{k}$ with a security parameter $k$, it is ensured to be secure against this attack.

\section{Conclusion}

A new type of key exchange protocol using polynomial maps is proposed. We proved the security of the protocol against key recovery attacks under the assumption of the hardness in solving a certain system of multivariate nonlinear polynomial equations with honest party setting. The flexibility on the choice of polynomial maps is demonstrated through a few examples. We showed a linear algebra attack which retrieves the secret polynomial map from the data in the communication channel and observed desired choices of parameters against this attack.

In a future investigation, it might be important to consider the intractability of the PME problem to determine appropriate parameter setting.

\section{Appendix A. Examples}

We illustrate simple examples for our key exchange protocol with small parameters $q=2, n=3, m=2$ and $d=1$. These examples are not secure since the parameters are too small, but we see how works the protocol.

\section{A.1. Example using an invertible polynomial map}

Below we give an example where $\psi$ is a triangular polynomial map.

(1) Alice takes an equation $f=0$ with $f=x+z \in$ $\mathbb{F}_{2}[x, y, z]$ and a solution $\underline{\sigma}=(0,0,0)$. Then, she sends $f$ to Bob.
(2) Bob takes $\underline{g}, \underline{\psi}, \underline{r} \in \mathbb{F}_{2}[x, y, z]$ given by

$$
\begin{aligned}
& \underline{g}=(x+y+1, x+y+z+1, x+1), \\
& \underline{\psi}=\left(x, y+x^{2}+x+1, z+x y+y^{2}+1\right), \\
& \underline{r}=(y+z+1, y+1, z+1)
\end{aligned}
$$

and computes $\underline{c}=\underline{\psi} \circ \underline{g}+f \underline{r}$ which yields

$$
\begin{gathered}
\underline{c}=\left(x y+x z+y z+y+z^{2}+z+1,\right. \\
\left.x^{2}+x y+x+y^{2}+y z, y z\right) .
\end{gathered}
$$

Then, he sends $\underline{c}$ and $g$ to Alice.

(3) Alice computes $\underline{u}=\underline{c}(\underline{\sigma})=(1,0,0), \underline{s}=g(\underline{\sigma})=$ $(1,1,1)$ and sends $\underline{u}$ to Bob.

(4) Since $\psi$ is a triangular polynomial map, it is invertible, hence

$$
\begin{aligned}
\underline{\psi}^{-1}= & \left(x, x^{2}+x+y+1, x^{4}+x^{3}+x y\right. \\
& \left.+x+y^{2}+z\right) .
\end{aligned}
$$

Bob then recovers $\underline{s}$ by $\underline{s}=\underline{\psi}^{-1}(\underline{u})=(1,1,1)$.

\section{A.2. Example using an HFE-based polynomial map}

We replace $\psi$ by an HFE-based polynomial map in Example A. $\overline{1}$.

Consider an irreducible polynomial $t^{3}+t+$ $1 \in \mathbb{F}_{2}[t]$. Let us take an extension $K=\mathbb{F}_{2}[t] /\left(t^{3}+\right.$ $t+1)$ over $\mathbb{F}_{2}$ of degree three. Then $\left\{1, t, t^{2}\right\}$, the set of three representatives $\bmod t^{3}+t+1$, forms a basis of $K$ over $\mathbb{F}_{2}$ giving an isomorphism $\phi(x, y, z)=x+y t+z t^{2}$ from $\mathbb{F}_{2}^{3}$ to $K$.

Suppose that Bob takes the polynomial $F(X)=$ $(1+t) X^{4}+X^{3}+(1+t) X^{2}+\left(1+t^{2}\right) X+1+t \in$ $K[X]$ which induces the following quadratic polynomial map (here we use the field equations $\left.x^{2}-x=y^{2}-y=z^{2}-z=0\right)$ :

$$
\begin{aligned}
\underline{\psi} & :=\phi^{-1} \circ F \circ \phi \\
& =(y z+1, x y+x z+1, x y+x+y) .
\end{aligned}
$$

Using the same $f, \underline{g}, \underline{r}$ as in Sec. A.1, Bob computes $\underline{c}=\underline{\psi} \circ \underline{g}+f \underline{r}:$

$$
\begin{aligned}
\underline{c}= & \left(x^{2}+x+y z+y+z^{2}, x z+x+y^{2}+y+1,\right. \\
& \left.x^{2}+x+y^{2}+y z+z^{2}+z+1\right) .
\end{aligned}
$$

Then Alice computes $\underline{u}=\underline{c}(\underline{\sigma})=(0,1,1), \underline{s}=$ $\underline{g}(\underline{\sigma})=(1,1,1)$ and sends $\underline{u}$ to Bob.

Now Bob computes the set $\left\{\underline{\psi}^{-1}(\underline{u})\right\}=\{(0,1,1)$, $(1,1,1)\}$ of candidates of the common key because 
the equation $F(X)-\phi(\underline{u})=(1+t)\left(X+t+t^{2}\right) \times$ $\left(X+1+t+t^{2}\right)\left(X^{2}+\left(1+t+t^{2}\right) X+t\right)=0$ has the solution

$$
\left\{t+t^{2}, 1+t+t^{2}\right\}=\{\phi(0,1,1), \phi(1,1,1)\} .
$$

Thanks to $\underline{g}^{-1}=(z+1, x+z, x+y)$, he can identify the true common key $(1,1,1)$ by verifying $f\left(\underline{g}^{-1}(0,1,1)\right)=f(0,1,1)=1 \neq 0 \quad$ and $\quad f\left(\underline{g}^{-1}(1\right.$, $1, \overline{1}))=f(0,0,0)=0$.

\section{Acknowledgments}

The authors are greatly indebted to the anonymous referees for giving valuable comments and suggestions that clarified the construction of the paper. The authors are also grateful to Doctor Ryo Fujita and Doctor Satsuya Ohata for their valuable advice regarding this investigation. This research was partly supported by JSPS Grant-in-Aid for Scientific Research (C), Grant Number 26520208.

\section{Authors' Contributions}

All the authors contributed original ideas for obtaining the results. All of them read and approved the final paper.

\section{Competing Interests}

The authors declare that they have no competing interests.

\section{Declarations}

\section{Ethics approval and consent to participate}

The authors declare that these are not applicable in this paper.

\section{Consent for publication}

The authors declare that this is not applicable in this paper.

\section{Availability of data and material}

The experiment in this paper is reproducible except the difference due to the pseudo random generations of the test instances.

\section{References}

1. K. Akiyama and N. Hirata-Kohno, A key exchange protocol using polynomial map (in Japanese), Symp. Cryptography and Information Security, 3A3-1 (The Institute of Electronics, Information and Communication Engineers, 2017).

2. M. R. Berlekamp, Factoring polynomials over finite fields, Bell Syst. Tech. J. 46 (1967) 1853-1859.

3. J. Ding, J. E. Gower and D. S. Schmidt, Multivariate Public Key Cryptosystems (Springer, 2006).

4. A. van den Essen, Polynomial Automorphisms and the Jacobian Conjecture, Progress in Mathematics, Vol. 190 (Birkhäuser, 2000).

5. M. R. Garey and D. S. Johnson, Computers and Intractability: A Guide to the Theory of NP-Completeness (W. H. Freeman, 1979).

6. N. Hirata-Kohno and A. Pethö, On a key exchange protocol based on Diophantine equations, Infocommun. J. 5(3) (2013) 17-21.

7. T. T. Moh, An application of algebraic geometry to encryption: Tame transformation method, Rev. Mat. Iberoamericana 19 (2003) 667-685.

8. W. M. Schmidt, Equations Over Finite Fields: An Elementary Approach (Kendrick Press, 2004).

9. H. Yosh, The key exchange cryptosystem used with higher order Diophantine equations, Int. J. Netw. Sec. Appl. 3 (2011) 43-50. 